\title{
Rechtsprechung
}

\section{Zur Mitteilungspflicht beim privaten Kunstverkauf}

\author{
Oberlandesgericht Karlsruhe, Urteil vom 15.10.2013 - 17 U 8/13
}

1. Eine (vor-)vertragliche Nebenpflicht des Verkäufers, ungefragt die Erwerbsumstände mitzuteilen, besteht bei Kaufvertragsverhandlungen von Privatpersonen grundsätzlich nicht, auch wenn sich diese auf ein Kunstobjekt (Gemälde) beziehen.

2. Ein kaufrechtliche Gewährleistung auslösender Mangel eines unsignierten Kunstobjekts (hier Gemälde von „Boudin“), für das eine Expertise vorgewiesen wird, liegt nicht bereits dann vor, wenn das Werk nicht im Werkverzeichnis für den Künstler enthalten ist, es sei denn die Vertragsparteien hätten eine solche Beschaffenheit bei Vertragsabschluss vereinbart.

\section{Zum Vorliegen von Arglist (hier nicht gegeben). (Amtliche Leitsätze)}

\section{Gründe}

I.

- Der Kläger nimmt den Beklagten nach dem Erwerb eines Gemäldes auf Schadensersatz in Anspruch.

Der Beklagte hatte verschiedene Gemälde, u.a. den streitbefangenen „Boudin“, über "eBay“ in der Kategorie "Antiquitäten \& Kunst > Malerei > Gemälde 1800-1899 > Originale der Zeit" zum Verkauf angeboten. Das vom Maler nicht signierte und nicht datierte Gemälde, das der Beklagte am 17. September $2008 \mathrm{im} \mathrm{M.} \mathrm{Auktionshaus} \mathrm{N.} \mathrm{zum} \mathrm{Preis} \mathrm{von} \mathrm{6.710} \mathrm{Euro} \mathrm{(5.500}$ Euro zuzüglich Aufgeld von 1.210 Euro) erworben hatte, war von ihm wie folgt beschrieben:

\section{"Ölgemälde Eugène Boudin mit Fotoexpertise}

\section{Juli 1824 Honfleur - 8. August 1898 in Deauville".}

Nach telefonischer Kontaktaufnahme traf sich der Kläger am 25. März 2009 mit dem Beklagten in dessen Wohnung in M. Er besichtigte das Bild und nahm Einsicht in die „Expertise" - ein handgeschriebenes Schriftstück des „E. Generaldirektors der Staatlichen Museen in Berlin Geheimrat Prof. Dr. H. Z." von Dezember 1961. Darin heißt es u.a.:

„Das umseitig wiedergegebene Ölbild auf Holz 34,5 x 47,3 cm habe ich im Original untersucht. Es ist eine Arbeit von Eugène Boudin (1824 - 1898), des bekanntesten Interpreten der Küstenlandschaft der Normandie und Bretagne in seiner Zeit. ...".

Nach einer Bedenkzeit, innerhalb derer der Kläger die Wohnung des Beklagten verlassen hatte, erwarb er nach Rückkehr noch am selben Tag das Bild zum Preis von 26.000 Euro. Die Parteien unterzeichneten ein mit "Quittung" bezeichnetes
Schriftstück. Darin heißt es u.a.: "Laut Foto-Expertise von Prof. Dr. Z. handelt es sich um ein original Werk des Malers Eugene Boudin".

Mit Schreiben vom 13. August 2009 erklärte der Kläger den Rücktritt vom Kaufvertrag, hilfsweise die Anfechtung wegen arglistiger Täuschung. Mit Schreiben vom 29. November 2011 erneuerte der Kläger die Anfechtung wegen arglistiger Täuschung. Hilfsweise erklärte er die Anfechtung seiner Vertragserklärung auch wegen Irrtums über eine verkehrswesentliche Eigenschaft.

Der Kläger hat behauptet, das Gemälde sei keine Arbeit von Eugène Boudin. Das Gemälde sei - insoweit unstreitig - nicht im Werkverzeichnis der Experten Robert und Manuel Schmit, Paris, aufgeführt. Manuel Schmit habe nach Begutachtung die Ansicht vertreten, das Bild sei kein Originalwerk von Eugène Boudin. Auch habe das Auktionshaus C. auf eine Anfrage seinerseits erklärt, das Gemälde sei für den internationalen Markt nicht geeignet.

Der Kläger hat ferner behauptet, im Falle der Echtheit des Gemäldes wäre dieses mindestens 120.000 Euro wert gewesen. Unter Abzug der Verkäuferprovision sowie der Versicherung in Höhe von $11,5 \%$ sei ihm daher ein Schaden von 106.200 Euro entstanden.

Er hat gemeint, das Werk sei mangelhaft, weil es ihm als echtes Gemälde von Eugène Boudin verkauft worden sei. Da der einzige anerkannte Experte Manuel Schmit das Bild als nicht von Eugène Boudin stammend einstufe, sei es als Arbeit von Eugène Boudin nicht verkäuflich. Dies stelle einen Mangel dar, der den Rücktritt des Klägers vom Kaufvertrag gerechtfertigt habe. Der Beklagte hafte auf das positive Interesse, weil er zumindest fahrlässig gehandelt habe. Der Beklagte habe angesichts des 
Umstands, dass er das Werk selbst etwa ein halbes Jahr vor dem Verkauf für 5.500 Euro zuzüglich Aufgeld (nur) „als Boudin zugeschrieben" erworben habe, gewusst, dass das Bild nicht echt sei.

Auch die Anfechtung des Kaufvertrags wegen arglistiger Täuschung greife durch. Der Beklagte habe ihm vor Vertragsabschluss nicht erklärt, dass er selbst nicht für die Echtheit des Bildes habe einstehen wollen. Er hätte den Kläger darauf hinweisen müssen, dass er selbst das Werk erst ein halbes Jahr vorher in einer Auktion zum Preis von 5.500 Euro (zuzüglich Aufgeld) "als Boudin zugeschrieben" erworben habe.

Der Beklagte hat bestritten, dass das streitbefangene Gemälde nicht von Eugène Boudin stammt. Ausweislich des im selbständigen Beweisverfahren eingeholten Gutachtens des Sachverständigen Prof. Dr. L. handele es sich bei dem Bild um ein von Eugène Boudin gemaltes Werk. Außerdem habe er sich auf die Expertise von Prof. Dr. H. Z. verlassen dürfen.

Das Landgericht hat der Klage nach Beweisaufnahme durch Einholung eines schriftlichen Gutachtens der Sachverständigen PD Dr. H. S. und unter Hinzuziehung des im selbständigen Beweisverfahren vor dem Landgericht Mannheim - $10 \mathrm{OH}$ 8/09 erstatteten Gutachtens in Höhe von 26.000 Euro (nebst Zinsen) stattgegeben und die Klage im Übrigen abgewiesen. Der Kläger habe lediglich Anspruch auf Rückzahlung des an den Beklagten gezahlten Kaufpreises von 26.000 Euro Zug um Zug gegen Rückgabe des streitgegenständlichen Bildes. Weitergehenden Schadensersatz könne er nicht beanspruchen. Insbesondere habe der Kläger keinen (auf dem Kaufvertrag beruhenden) Schadensersatzanspruch gemäß §§ 434, 437 Nr. 3, 440, 281 BGB, da nach der durchgeführten Beweisaufnahme nicht zur Überzeugung des erkennenden Gerichts bewiesen sei, dass es sich bei dem Bild nicht um einen echten Boudin handele, das Gemälde also nicht von Eugène Boudin stamme und deshalb mangelhaft sei.

Allerdings könne der Kläger unter dem Gesichtspunkt der culpa in contrahendo gemäß § 280 BGB in Verbindung mit § 311 Abs. 2 Nr. 1 BGB die Erstattung des Kaufpreises Zug um Zug gegen Rückgabe des Gemäldes beanspruchen. Zwar sei nach Gefahrübergang grundsätzlich von einem Vorrang der Gewährleistungsvorschriften vor den Regeln des Verschuldens bei Vertragsschluss auszugehen. Eine Ausnahme bestehe allerdings dann, wenn von einem arglistigen Verhalten des Verkäufers bei Vertragsschluss auszugehen sei. Dies sei hier der Fall.

Dagegen wenden sich beide Parteien mit der Berufung. Der Kläger verfolgt den erstinstanzlich geltend gemachten Anspruch in vollem Umfang weiter, soweit seine Klage abgewiesen worden ist, während der Beklagte weiterhin die vollständige Abweisung der Klage erstrebt. Das verkaufte Bild sei echt, wie die Beweisaufnahme ergeben habe. Eine Offenbarungsverpflichtung zur Provenienz und hinsichtlich des von inm gezahlten Ankaufspreises habe nicht bestanden. Der Kläger habe auch nie nach dem Ankaufspreis gefragt. Im Übrigen sei das Bild mit der Expertise von Prof. Dr. Z. als „zugeschrieben“ verkauft worden. Von einer arglistigen Täuschung des Beklagten könne nicht ausgegangen werden.

Demgegenüber hält der Kläger daran fest, dass das vom Beklagten erworbene Gemälde nicht von Boudin stamme. Insoweit sei maßgeblich und allein auf die Auffassung des Verfassers des Werkverzeichnisses von Boudin, Manuel Schmit, Paris, abzustellen, der die Aufnahme in das Werkverzeichnis mit der Begründung ablehne, das Gemälde sei nicht von Boudin gemalt worden, dieses also für nicht echt halte. Das Bild sei daher auf dem Kunstmarkt praktisch unverkäuflich.

Auch im Berufungsrechtszug beruft sich der Kläger auf Einholung eines weiteren Sachverständigengutachtens mit Blick auf die Einschätzung von Manuel Schmit, der eine sehr große Materialkenntnis schon allein aufgrund der Bandbreite der von inm überprüften Bilder habe, und benennt, wie bereits in erster Instanz, Dr. R. K., Z. Erstmals im Berufungsrechtszug schlägt der Kläger weiter als Sachverständige vor A.-M. B., die Direktorin des Eugène-Boudin-Museums in $\mathrm{H}$., die ebenfalls eine ausgewiesene Kennerin des Werks von Boudin sei, weil sie in ihren Museumsbeständen hunderte von Werken Eugène Boudins habe und selbst Kunsthistorikerin sei.

Der Kläger macht ferner geltend, die fehlende Anerkennung des Bildes als echt durch den Herausgeber des Werkverzeichnisses sei hier ein Fehler des Gemäldes an sich, weil der Beklagte es als Arbeit von Boudin verkauft habe. Jedenfalls habe das Landgericht seinem Hilfsantrag auf Einholung eines Obergutachtens durch Dr. R. K., der eine umfassende Ausstellung der Stiftung L. mit Werken Boudins organisiert und zu der Ausstellung einen Katalog mit Biografie herausgegeben habe, stattgeben müssen.

Die Parteien treten jeweils der Berufung der Gegenseite entgegen und beantragen deren Zurückweisung.

II.

Die zulässige Berufung des Klägers ist in der Sache nicht gerechtfertigt, während die ebenfalls zulässige Berufung des Beklagten Erfolg hat und zur vollständigen Abweisung der Klage führt. Ein Anspruch des Klägers auf Rückabwicklung des Kaufs oder Schadensersatz gegen den Beklagten besteht nicht. Entgegen der Auffassung des Landgerichts, vermag der Senat ein arglistiges Verhalten des Beklagten im Rahmen der Vertragsverhandlungen bis zum Vertragsabschluss nicht zu erkennen. Das Landgericht verweist selbst auf die dem Bild beiliegende Expertise von Prof. Dr. H. Z., nach der das Bild tatsächlich von Boudin stammt, wie er ausdrücklich ausführt. Auf diese Information durfte sich der Beklagte verlassen, zumal das Gemälde nach den tatsächlichen Feststellungen des Landgerichts aufgrund der durchgeführten umfangreichen Beweisaufnahme in der zweiten Hälfte des 19. Jahrhunderts gefertigt worden ist und das Landgericht auf der Grundlage des Gutachtens des Sachverständigen Prof. Dr. L. die Echtheit des Werks nicht in Zweifel 
zieht. Danach hat der Kläger den Nachweis, das streitbefangene Gemälde stamme nicht von dem Maler Eugène Boudin, nicht geführt. Anders als dies das Landgericht gesehen hat, musste der Beklagte den Kläger nicht von sich aus darauf hinweisen, unter welchen Umständen und zu welchem Preis er das Gemälde erworben hat. Der Kläger hatte danach nicht gefragt. Eine solche Nebenpflicht, ungefragt die Erwerbsumstände mitzuteilen, lässt sich für Kaufvertragsverhandlungen zwischen Privatpersonen, auch wenn es um ein Kunstobjekt (Gemälde) geht, nicht begründen. Die Verhandelnden haben dabei offensichtlich jeweils ihr eigenes Interesse im Auge. Das Landgericht hat hierbei auch insbesondere dem Umstand, dass das Werk unsigniert und undatiert ist, nicht die notwendige Beachtung geschenkt.

\section{Berufung des Klägers}

1. Zutreffend führt das Landgericht in den Entscheidungsgründen aus, dass dem Kläger kein Schadensersatzanspruch gemäß $\S \S 434,437$ Nr. 3, 440, 281 BGB zusteht, weil ein Mangel des Bildes nicht vorliegt. Nach den tatsächlichen Feststellungen des Landgerichts, an die das Berufungsgericht gemäß $\S 529$ Abs. 1 Nr. 1 ZPO gebunden ist, hat der Kläger die Unechtheit des Gemäldes, das nach der vertraglich vorausgesetzten Beschaffenheit von Eugène Boudin stammt, nicht bewiesen. Die durchgeführte Beweisaufnahme hat nicht zur Überzeugung des Landgerichts geführt, dass das streitbefangene Gemälde nicht von Eugène Boudin stammen könnte.

Die hiergegen gerichteten Angriffe des Klägers sind unbegründet. Der Kläger vermag keine solchen konkreten Zweifel aufzuzeigen, die eine erneute Feststellung durch das Berufungsgericht oder die Einholung eines weiteren Sachverständigengutachtens gemäß $\S 412$ Abs. 1 ZPO gebieten würden oder angezeigt erscheinen ließen. Die Beweiswürdigung im angefochtenen Urteil ist nicht zu beanstanden. Aus den Entscheidungsgründen wird deutlich, dass sich das Landgericht aufgrund der Ausführungen des vom Gericht beauftragten Sachverständigen Prof. Dr. C. L. (§ 493 Abs. 1 ZPO), der das Gemälde für ein Werk von Eugène Boudin hält, das dieser höchstwahrscheinlich in den siebziger Jahren in Berck geschaffen habe, nicht hat davon überzeugen können, dass das streitbefangene Gemälde nicht von Boudin gemalt ist. Insbesondere hat es der Sachverständige nicht für ungewöhnlich gehalten, dass das Gemälde bisher nicht bekannt geworden ist. Mit weiteren, bisher unbekannten Werken Boudins müsse gerechnet werden, worauf auch die beiden Supplementbände von Robert und Manuel Schmit hindeuteten. Schließlich lasse die fehlende Signatur bei dem hier zu begutachtenden Bild, die sich Boudin vielleicht für den Verkauf vorbehalten habe, weder Zweifel an seiner Eigenhändigkeit aufkommen, noch deute sie auf ein mehr oder minder geringes Maß der Vollendung hin. Es handele sich um ein bildmäßig vollendetes Gemälde.
In seiner weiteren Stellungnahme vom 9. März 2011 weist der Sachverständige ferner darauf hin, dass sich dichte pastose Malweise im Werk Boudins durchaus finde. Mit einer allgemeineren Bemerkung und weiteren Erläuterungen betont der Sachverständige Prof. Dr. L., dass - auch wenn die Lage hinsichtlich der Vergleichsbeispiele gerade für die in Berck entstandenen Werke schwierig sei - die gemeinsamen Züge derart deutlich seien, dass er keinen Grund habe, das Bild als ein originales Werk von Boudin zu bezweifeln. Ein Fälscher hätte sich wohl ein attraktiveres Motiv gewählt und sicher die Signatur hinzugefügt.

Auch die Sachverständige PD Dr. H. S. beschreibt das Bild in seiner Ausführung als nass-in-nass und rasch mit skizzenhaftem Charakter und als variantenreich sowohl im Hinblick auf Pinselduktus und Pastosität als auch Farbigkeit.

Die vom Kläger vorgebrachten Einwendungen, die er auf die negative Bewertung von Manuel Schmit stützt, hat der gerichtliche Sachverständige zur Kenntnis genommen. Er hat überzeugend begründet, weshalb er die Einschätzung Schmits nicht teilt. Auf seine schriftlichen Gutachten und die Ausführungen im Rahmen der mündlichen Erläuterung der Gutachten vor dem Landgericht am 10. März 2011 wird verwiesen. Insbesondere erläuterte er, man könne nicht davon sprechen, dass es sich insgesamt um eine schwerfällige und pastose Malweise handele. Nicht alle Bereiche des Bildes seien in derselben Malweise geschaffen. Ferner konnte der Sachverständige nicht bestätigen, dass für das Werk Boudins ein lebhafter Pinselstrich charakteristisch sei. Diesen Pinselstrich gebe es in einem Teil des Werks, aber nicht insgesamt. Das Schaffen Boudins weise insgesamt eine sehr große Unterschiedlichkeit auf. Auch die dichte und pastose Malweise, die als Kritikpunkt gegen das streitgegenständliche Werk vorgebracht werde, gebe es im Werk von Boudin.

Auch erbrachte das im Rechtsstreit zusätzlich eingeholte Gutachten der Sachverständigen PD Dr. H. S. vom D. Institut keine Anhaltspunkte dafür, dass das Werk aus maltechnisch/ naturwissenschaftlicher Sicht nicht von Boudin stammen könnte. Insbesondere steht nach den Untersuchungen der Sachverständigen fest, dass das Werk in der zweiten Hälfte des 19. Jahrhunderts gefertigt worden ist. Die Ergebnisse der Infrarotreflektographie sprechen danach gegen das Vorliegen einer bloßen Kopie. Dort finden sich - so die Ausführungen der Sachverständigen - Anzeichen, die charakteristisch sind für den künstlerischen Prozess einer Kompositionsfindung. Neben den in der Infrarotaufnahme sichtbaren Änderungen der Bildkomposition sei aber auch die freie und variantenreiche Malweise typisch für einen originären Werksprozess und lasse ausschließen, dass es sich hier um eine reine "Kopistenarbeit" handele.

Die Einholung eines weiteren Gutachtens nach § 412 Abs. 1 ZPO erschien nicht erforderlich oder sonst angezeigt. Der Sachverständige Prof. Dr. L. hat sich eingehend mit den Werken, die gesichert von Boudin stammen, befasst und dies, wie die erstinstanzlich erkennende Richterin, die auch im selbständigen 
Beweisverfahren tätig war, in den Entscheidungsgründen ausführt, anschaulich bei seiner mündlichen Anhörung hinsichtlich Malweise und Motiven dem Gericht erläutert. Eine überlegene Forschungsmethode oder erheblich breitere Erfahrung der beiden vom Kläger weiter benannten Gutachter ist nicht schon deshalb anzunehmen, weil diese eine größere Anzahl von Originalwerken Boudins gesehen haben und möglicherweise einen unmittelbaren Vergleich mit vorhandenen Werken in einem Museum vornehmen könnten. Der Kläger hat den vom Gericht beauftragten Sachverständigen Prof. Dr. L. auf Empfehlung von Dr. G. C. benannt und selbst bereits umfangreich mit Dr. R. K. korrespondiert und eine vorläufige Einschätzung von ihm erreicht, ohne dass dieser jedoch das Original gesehen hätte. Eine Begutachtung durch ihn kommt danach nicht mehr näher in Betracht.

Die vom Beklagtenvertreter im Senatstermin übergebenen Auszüge aus dem Internet zeigen die breite Erfahrung des gerichtlichen Sachverständigen Prof. Dr. L. auf, wie sie sich auch in dessen fundierter Begutachtung in den schriftlichen Gutachten und der mündlichen Erläuterung zeigt, auf die sich der Senat mit dem Landgericht bei seiner Entscheidung stützt und die in jeder Hinsicht ausreichend erscheinen.

Damit hat der Kläger den Nachweis eines Mangels des Gemäldes, weil es nicht von Boudin stamme, nicht geführt.

Von einem Mangel des Kaufobjekts ist auch nicht deshalb auszugehen, weil das Gemälde (nur) Eugène Boudin „zugeschrieben" ist, aber - was der Kläger schon als Mangel ansieht - nicht "unzweifelhaft echt" sei, weil Manuel Schmit als Verfasser des Werkverzeichnisses von Eugène Boudin das Bild nicht als echt anerkenne. Denn eine solche Beschaffenheit haben die Parteien bei Vertragsabschluss gerade nicht vereinbart. Es kommt daher nicht darauf an, ob der Beklagte, wie er unter Beweisantritt vorträgt, das Bild ausdrücklich nur als aufgrund der Expertise von Prof. Dr. H. Z. Eugène Boudin „zugeschrieben“ an den Kläger verkauft hat.

Das streitbefangene Gemälde ist vom Künstler weder signiert noch datiert. Die Person desjenigen, der das Werk gefertigt hat, lässt sich somit nicht unmittelbar am Werk ablesen, sondern von vornherein nur durch einen Kunstsachverständigen feststellen, der das Werk nach allen seinen Charakteristika bewertet und einem Maler zuordnet. Genau dies ist durch die Expertise von Prof. Dr. Z. geschehen, die Gegenstand der Vertragsverhandlungen der Parteien gewesen ist. Weder ist die Beschaffenheit des Bildes in Bezug auf das Werkverzeichnis für die Werke von Eugène Boudin näher konkretisiert worden noch gibt es dazu eine Erklärung oder Garantie des Beklagten. Dies behauptet auch der Kläger nicht. In einem solchen Fall hätte das Werk konkret dahin beschrieben werden müssen, dass es in einem bestehenden Werkverzeichnis oder in einem Katalog aufgenommen ist oder von einem bestimmten Experten anerkannt wird. Die Parteien haben dazu keine Erklärungen von ihrem Vertragspartner verlangt oder selbst abgegeben; der Klä- ger trägt hierfür nichts vor. Es ist auch keine Verzeichnisnummer o.ä. angegeben worden, die auf einen Katalog Bezug genommen oder hingewiesen hätte. Schließlich zeigt der Kläger auch nicht auf, dass das Bild bereits vor Vertragsabschluss dem Herausgeber des Werkverzeichnisses Schmit einmal vorgelegen hätte oder gar von ihm als unecht bewertet worden wäre und der Beklagte davon Kenntnis gehabt hätte. Es gibt auch keine Anhaltspunkte dafür, dass die Parteien im Zeitpunkt des Vertragsabschlusses Manuel Schmit als einzigen oder den Experten angesehen hätten, auf dessen Beurteilung der Echtheit des Gemäldes es allein ankommen solle.

2. Eine Pflicht des Beklagten, die Umstände des Ankaufs ungefragt offenzulegen, bestand nicht. Insbesondere ist auch kein arglistiges Verhalten darin zu sehen, dass der Beklagte das Werk nicht mit dem ausdrücklichen Hinweis darauf beschrieben hat, dass das Gemälde (nur) nach einer Expertise von Prof. Dr. H. Z. Eugène Boudin zugeschrieben wird und - so wird man die Auffassung des Klägers weiter zusammenfassen können - es keinen unzweifelhaften Beweis für den Maler des Werks gibt. Zu einer solchen näheren Erläuterung seines Verkaufsangebots war der Beklagte nicht verpflichtet. Dies konnte der Kläger bereits anhand der Umstände des Kaufs von selbst erkennen. Denn ihm wurde zu dem unsignierten und undatierten Gemälde, das seinen Urheber nicht unmittelbar erkennen lässt, lediglich die Expertise von Prof. Dr. Z. vorgewiesen und - dessen Einschätzung folgend - das Gemälde als von Eugène Boudin gemalt bezeichnet.

Dass der Beklagte Zweifel an der Expertise oder dem "Ruf" von Prof. Z. (insoweit sollen nach dem Sachverständigen bei seiner mündlichen Anhörung am 10. März 2011 Gutachten in diesen Bereichen früher anders erstellt worden sein, als dies vielfach heute der Fall sei, und müssten durch ein ausführlicheres Gutachten ergänzt werden, um auch heute noch als anerkanntes Gutachten bezeichnet werden zu können) gehabt hätte, ist weder substantiiert dargetan noch unter Beweis gestellt. Der Beklagte durfte sich darauf verlassen, dass dessen Expertise zutrifft. Jedenfalls handelte er insoweit nicht arglistig, zumal das Ergebnis der Expertise von Prof. Dr. Z. ja auch im Gerichtsverfahren durch die eingeholten Sachverständigengutachten bestätigt ist oder gestützt wird. Es gibt auch keine hinreichenden Anhaltspunkte dafür, dass der Beklagte bei Vertragsabschluss gewusst hätte, dass es ein von der Galerie Schmit in Paris geführtes Werkverzeichnis für Boudin gibt, in dem das Kaufobjekt nicht aufgeführt ist, und dass der Kunstmarkt deshalb für die Beurteilung, ob das Werk echt und von Boudin gemalt ist, besonderen Wert auf die Einschätzung von Manuel Schmit legen wird.

Der Kläger kann sich insbesondere auch nicht auf den von ihm bezahlten Kaufpreis stützen, um seine Behauptung weiter zu untermauern, der Beklagte habe das Werk als nachweislich echt zum Verkauf gestellt. Nach dem Vorbringen des Klägers selbst zur Schadenshöhe werden für vergleichbare Werke von Eugène Boudin, die von Manuel Schmit anerkannt sind, Preise 
von deutlich über 100.000 Euro erzielt. Vor diesem Hintergrund lässt der vom Kläger bezahlte Preis von 26.000 Euro keine Interpretation dahin zu, der Beklagte habe dafür einstehen wollen, das Bild sei unter allen Umständen im Kunsthandel jederzeit als Werk von Boudin erfolgreich zu veräußern, und er garantiere dies. Es gibt auch keine Garantie des Beklagten dafür, dass der Kläger das Bild kurzfristig mit einem Gewinn von 80.000 Euro über ein Auktionshaus versteigern kann oder dass eine Anerkennung durch den Herausgeber des Werkverzeichnisses von Boudin, so es eines gebe, gesichert ist. Im Rahmen der Vertragsverhandlungen war stets nur die Expertise von Prof. Dr. Z. angesprochen.

Schließlich ist ergänzend festzuhalten, dass es auch keine Anhaltspunkte dafür gibt, der Beklagte hätte für ein lediglich durch die Expertise von Prof. Z. Boudin zugeschriebenes Werk einen sittenwidrig überhöhten Kaufpreis verlangt, zumal auch der gerichtlich beauftragte Sachverständige Prof. Dr. L. seine Überzeugung davon, dass das Gemälde aus der Hand von Boudin stammt, nachvollziehbar und plausibel dargelegt hat und die maltechnisch-naturwissenschaftliche Untersuchung von Privatdozentin Dr. H. S. dies stützt, dem Gutachten von Prof. Dr. C. L. jedenfalls nicht entgegensteht.

\section{Berufung des Beklagten}

Ansprüche des Klägers gegen den Beklagten im Zusammenhang mit dem Erwerb des Gemäldes bestehen daher insgesamt nicht. Bei dieser Sachlage war - neben der Zurückweisung der Berufung des Klägers - auf die Berufung des Beklagten das Urteil des Landgerichts, soweit es der Klage stattgegeben hat, zu ändern und die Klage insgesamt abzuweisen. (Entscheidung von der Redaktion bearbeitet.)

\section{Der Museumsbegriff im Umsatzsteuerrecht}

Oberverwaltungsgericht Nordrhein-Westfalen, Urteil vom 31. Juli 2013 - 14 A 457/13

Eine Sammlung ist für ein Museum ein Einrichtungs- und kein Aufgabenmerkmal. Zur Bestimmung des Museumsbegriffs nach \$ 4 Nr. 20 a USt G kann es daher dahinstehen, ob eine eigene oder ständige Sammlung vorliegt, solange gleiche kulturelle Aufgaben wie von Museen in öffentlich-rechtlicher Trägerschaft erfüllt werden. (Leitsatz der Redaktion)

\section{Tatbestand}

- Die Klägerin betreibt die am 19. Juni 1992 eröffnete Kunstund Ausstellungshalle der Bundesrepublik Deutschland - KAH - in Bonn.

In $\S 2$ Abs. 1 des Gesellschaftsvertrages wird der Gegenstand der Gesellschaft u.a. wie folgt definiert:

Gegenstand des Unternehmens ist es, die Kunst- und Ausstellungshalle der Bundesrepublik Deutschland zu betreiben und geistige und kulturelle Entwicklungen von nationaler und internationaler Bedeutung sichtbar zu machen, insbesondere durch Ausstellungen, die von der Gesellschaft veranstaltet oder - auch im Austausch - übernommen werden.

Auf Antrag der Klägerin bescheinigte die Bezirksregierung Köln mit Bescheid vom 6. März 1995, dass die KAH die gleichen kulturellen Aufgaben erfüllt wie die in $\S 4 \mathrm{Nr}$. 20 Buchst. a) S. 2 des Umsatzsteuergesetzes - UStG - genannten staatlichen und kommunalen Einrichtungen. Die Bescheinigung enthielt den Zusatz: „Sie wird auf Widerruf erteilt".

Mit Schreiben vom 4. März 2009 stellte die Klägerin den Antrag, die Bescheinigung zu widerrufen. Zur Begründung führte sie im Wesentlichen aus: Die Klägerin sei kein Museum, denn sie verfüge weder über eine wissenschaftliche Sammlung noch über eine Kunstsammlung. Die Ausstellungen würden gemeinsam mit Künstlern, Galeristen und privaten Leihgebern konzipiert und durchgeführt, wobei Leihgaben von in- und ausländischen Leihgebern für die Dauer der Ausstellung ausgeliehen würden. Darüber hinaus konzipiere und projektiere die Klägerin Tourneeveranstaltungen für verschiedene Ausstellungen, d.h. die von der Klägerin erarbeiteten Ausstellungskonzepte würden auch in anderen vergleichbaren Ausstellungshäusern gezeigt. Sie erfülle daher keineswegs die gleichen kulturellen Aufgaben wie die in $\S 4 \mathrm{Nr}$. 20 Buchst. a) UStG genannten staatlichen oder kommunalen Einrichtungen. Die Gleichstellung der Klägerin mit diesen Einrichtungen führe zu einem erheblichen 